\title{
Torsion of the Vermiform Appendix Secondary to Mucinous Cystadenoma
}

\author{
Sundus Abbasia, ${ }^{\mathrm{a}}$, Miguel Lopez-Viego ${ }^{\mathrm{b}}$
}

\begin{abstract}
Torsion of the vermiform appendix (TMA) is a rare entity that is often clinically indistinguishable from acute appendicitis. Primary torsion results from anatomical abnormalities and secondary torsion results from obstructive pathologic processes such as mucinous cystadenomas. Torsion secondary to a mucinous cystadenoma is found in $0.2-$ $0.3 \%$ of appendectomies. The diagnosis of TVA is difficult, requiring histological confirmation after surgical intervention. We present a rare case of TVA in a 35-year-old woman with abdominal pain for 2 days.
\end{abstract}

Keywords: Torsion; Vermiform appendix; Mucinous cystadenoma

\section{Introduction}

Acute appendicitis is an inflammatory condition and the most common cause of emergency abdominal surgery. Torsion of the vermiform appendix (TVA) is rare with only 35 cases reported $[1,2]$. Additionally, the clinical presentation mirrors acute appendicitis, making the preoperative diagnosis even more difficult. An appendectomy is necessary to make a definitive diagnosis [2]. We present an unusual case of a 35-year-old woman with abdominal pain suspicious for both gastrointestinal and gynecological etiologies. Initial diagnostic workup, including radiographic imaging, was indeterminate, leading clinical suspicion to guide treatment.

\section{Case Report}

A 35-year-old previously healthy woman presented to the emergency department of our teaching hospital with a chief

Manuscript submitted December 23, 2018, accepted January 3, 2019

aDepartment of Surgery, Saint Joseph's Regional Medical Center, Paterson, NJ, USA

bepartment of Surgery, Charles E. Schmidt College of Medicine, Florida Atlantic University, Boynton Beach, FL, USA

${ }^{\mathrm{c} C}$ Corresponding Author: Sundus Abbasi, Department of Surgery, Saint Joseph's Regional Medical Center, Paterson, NJ 07901, USA.

Email: Sundus.abbasi@gmail.com

doi: https://doi.org/10.14740/jcs358 complaint of epigastric pain that radiated to her pelvis for 2-day duration. The pain was sharp. She had associated nausea, but denied vomiting, diarrhea, or constipation. She is a G1P1001 and compliant with her oral contraceptives. Her past medical history was significant for controlled epilepsy. She had no past surgical history. She did not smoke and drinks occasionally. Her family history was non-contributory.

On presentation she was afebrile, with a blood pressure of $114 / 88$, pulse of 98 beats per minute, respiratory rate of 16 breaths per minute, and an oxygen saturation of $98 \%$ on room air. Her physical exam was significant for mild abdominal distention and tenderness to palpation in both lower quadrants, more profound in the right lower quadrant and suprapubic regions with focal guarding in her right lower quadrant.

Basic laboratory studies revealed mild hyponatremia (134 $\mathrm{mmol} / \mathrm{L}$ ) and an elevated serum creatinine (1.30 units) (110). A liver function panel was within normal limits. Complete blood count revealed elevated white blood cell count (16.6 units) with a left shift. A pregnancy test was negative. Transvaginal ultrasound revealed a complex, prominently cystic $8.6-\mathrm{cm}$ mass between the uterus and dome of the bladder, which appeared to be distinct from the ovaries. Computed tomography (CT) of the abdomen without contrast revealed an inadequately visualized appendix and a midline pelvic cystic structure measuring $6.7 \times 4.4 \mathrm{~cm}$ possibly arising from the appendiceal tip, and free pelvic fluid adjacent to the cecum (Fig. 1a-d).

A laparoscopic appendectomy was performed and revealed torsion of an appendiceal mucocele with gangrene of the appendix. Final pathology confirmed a markedly distended appendix containing a mucinous cystadenoma with necrosis and hemorrhage.

Her postoperative course was uneventful and she was discharged on the second postoperative day tolerating a regular diet.

\section{Discussion}

TVA is a rare condition that is typically clinically identical to acute appendicitis. Both conditions classically present with nausea, vomiting, and diffuse abdominal pain that localizes to the right lower quadrant, low-grade fever, and an elevated leukocyte count. A review of literature has revealed only nine cases of TVA associated with appendiceal mucinous neoplasms [3]. Primary TVA results from anatomical variations including a long appendix with a narrow stump or fan-shaped mesentery [4]. Etiologies of secondary TVA include mucinous 


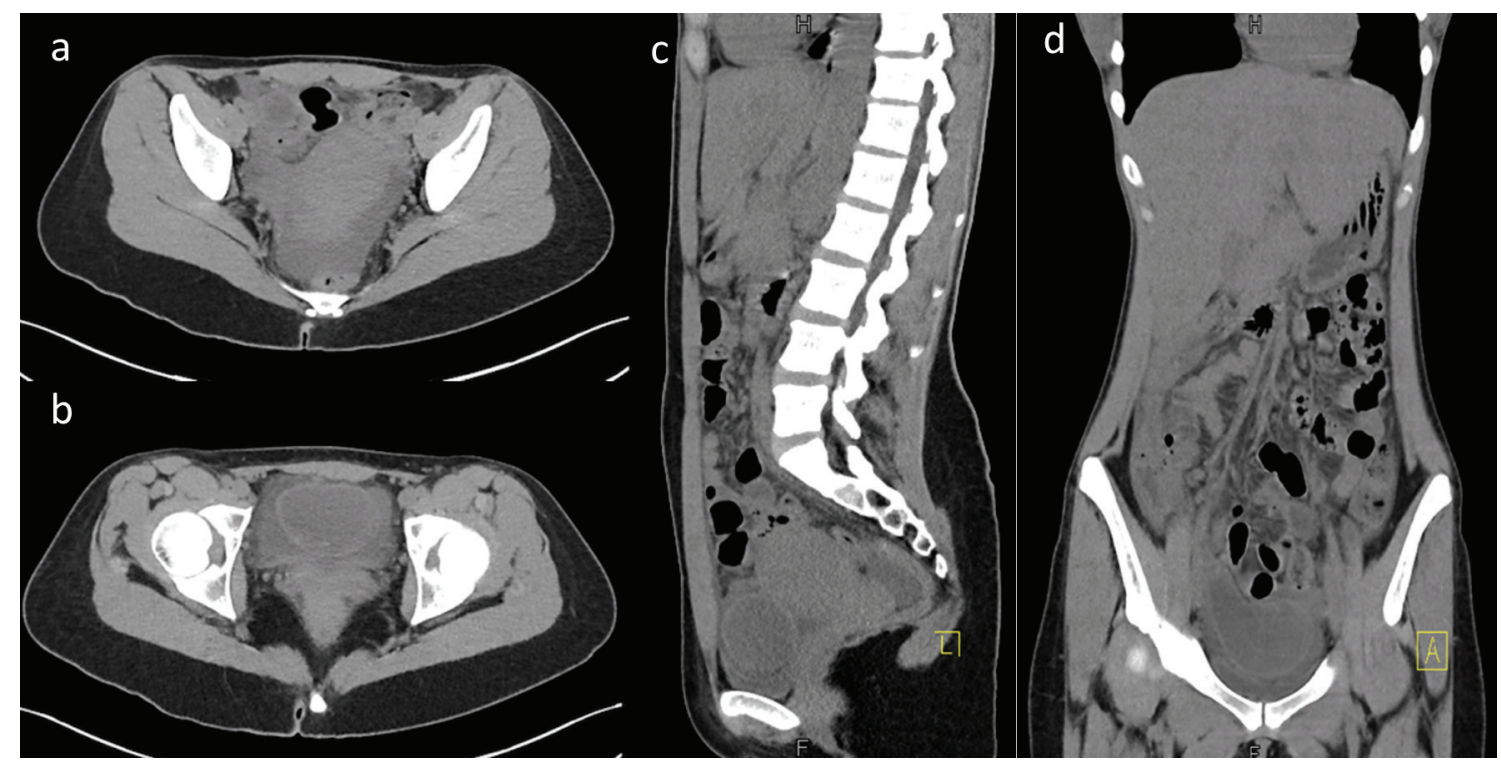

Figure 1. Axial CT view (a, b), sagittal CT view (c) and coronal CT view (d). All demonstrate a midline pelvic cystic structure possibly arising from the appendiceal tip and free pelvic fluid adjacent to the cecum.

cystadeomas, mucoceles, and fecaliths [5]. Specifically, mucous adenomas cause an overproduction of mucous leading to the formation of mucoceles, which invade into the appendiceal wall provoking inflammation [6]. The most common location for torsion is $1 \mathrm{~cm}$ from the appendiceal base in a counterclockwise rotation [7]. In adults, TVA is more common in men than in women with a 4.5:1 ratio [7].

Interestingly, this case involved the use of $\mathrm{CT}$ without contrast, which may have led to poor visualization of the appendix. In order to expedite future diagnosis and treatment, CT with contrast may be more useful to visualize blood flow and inflammatory changes. Additionally, CT with contract can reveal an enhancing cyst possibly aiding in the preoperative diagnosis of a mucocele or cystadenoma [4]. Research has suggested MRI as an alternative diagnostic tool to evaluate unknown lesions of the appendix, primarily for younger patients requiring diagnostic imaging when ultrasound is not confirmatory and in pregnant women where CT scanning is undesirable [8].

Clinical presentation of acute appendicitis and TVA are indistinguishable without confirmation from pathology. Appendectomy remains the standard of treatment for TVA. The patient's clinical presentation and imaging suggested either a gynecological or gastrointestinal source. Due to the close proximity of the structure to the ovaries, it was difficult to distinguish from gynecological pathology. Ultimately, the consequences of untreated TVA, such as rupture and generalized pseudomyxoma peritonei, warrant surgical intervention [6]. Laparoscopic appendectomy allowed for adequate visualization of the gangrenous appendix, excluding a gynecological origin.

\section{Conclusions}

While acute appendicitis is a very common surgical emergen- cy, secondary TVA with mucinous cystadenoma is rare. Differentiating between both conditions will continue to be difficult prior to appendectomy. This case highlights the importance of including TVA in the differential diagnosis of a patient with abdominal pain and a mass in the pelvis with equivocal imaging.

\section{References}

1. Woodruff R, McDonald JR. Benign and malignant cystic tumors of the appendix. Surg Gynecol Obstet. 1940;71:750-755.

2. Marsdin EL, Griffiths C. Primary torsion of vermiform appendix mimicking acute appendicitis. BMJ Case Rep. 2011;2011.

3. Mishin I, Ghidirim G, Zastavnitsky G, Popa C. Torsion of an appendiceal mucinous cystadenoma. Report of a case and review of literature. Ann Ital Chir. 2012;83(1):75-78.

4. Kitagawa M, Kotani T, Yamano T, Tsurudome H, Hatakeyama T, Kuriu Y, Nishi H, et al. Secondary torsion of vermiform appendix with mucinous cystadenoma. Case Rep Gastroenterol. 2007;1(1):32-37.

5. Wani I, Kitagawa M, Rather M, Singh J, Bhat G, Nazir M. Torsion of vermiform appendix with fecalith: a case report. Cases J. 2008;1(1):20.

6. Deans GT, Spence RA. Neoplastic lesions of the appendix. Br J Surg. 1995;82(3):299-306.

7. Val-Bernal JF, Gonzalez-Vela C, Garijo MF. Primary acute torsion of the vermiform appendix. Pediatr Pathol Lab Med. 1996;16(4):655-661.

8. Stark C, Jousi M, Enholm B. Preoperative assessment and treatment of appendiceal mucocele complicated by acute torsion: a case report. BMC Res Notes. 2014;7:1. 